\title{
Smart Tales: An Awareness Game for Ambient Assisted Living
}

\author{
Paolo Sernani ${ }^{1}$, Fabiano Dalpiaz ${ }^{2}$, Aldo Franco Dragoni ${ }^{1}$, Sjaak Brinkkemper $^{2}$ \\ 1 Dipartimento di Ingegneria dell'Informazione \\ Università Politecnica delle Marche, Italy \\ 2 Utrecht University, the Netherlands \\ f.dalpiaz@uu.nl, s.brinkkemper@uu.nl
}

\begin{abstract}
Despite the progress in ambient assisted living (AAL), the general audience is still mostly unaware of this term as well as of its purpose, enabling technologies, and potential. As a consequence, there are often misconceptions about AAL and smart homes, and the acceptance of AAL technologies is still too low. To cope with these problems, this paper presents a publicly available awareness game called Smart Tales, whose goal is to enhance the familiarity of its players with the notion and core concepts of AAL. In Smart Tales, the player has the role of an assisted patient in a smart home, and gets to learn about AAL and its technologies while trying to cheat the sensors that are placed in the house. In addition to presenting the design of the game following the Serious Games Design Assessment framework from the literature, we present results on engagement and learning that we obtained through a formative evaluation with ten users.
\end{abstract}

\section{Introduction}

The population ageing phenomenon [16] is expected to significantly increase the expenditures in healthcare [31] to assist elderly people all over the world. In this context, solutions are necessary to reduce these expenses, including the onerous costs for hospitalizing non-autonomous yet healthy elderlies. Ambient Assisted Living (AAL) comes to the rescue; its objective is to extend the time people can live in their preferred environment by increasing their autonomy, self-confidence and mobility [28].

Despite its promises, the acceptance of AAL and its technology is still unsatisfactory, and studies have shown a rather low intention of prospective users to adopt AAL techniques [2]. The reasons for low acceptance are many [14], and include technological concerns about the trustworthiness of AAL solutions [27] (in terms of functionality, reliability, usability) as well as ethical considerations such as the potential loss of privacy $[2,4]$, dignity [8] and autonomy [30].

Our diagnosis is that low acceptance is often caused by the lack of awareness of the purpose of AAL and its enabling technologies. Although this is not the only cause, we hypothesize that raising awareness could positively contribute to acceptance by avoiding misconceptions and by mitigating unjustified fears.

In this paper, we advocate the use of awareness games as a tool to deliver knowledge on the objectives and working principles of AAL. Awareness games are serious 
games [29] that aim at increasing awareness in a certain target audience about a given domain, in addition to providing engagement as a regular (video-)game. Awareness games have been proposed for several domains, including environmental issues [10], security engineering [21], and flooding policies [23].

Specifically, we present our developed game called Smart Tales, where the player impersonates an assisted patient living in a smart home, and gets to learn about AAL and its technologies while trying to cheat the sensors that are placed in the house. While doing so, the player collects informative cards concerning AAL, which are necessary to answer the knowledge quizzes at the end of each level so as to unlock further levels.

We make three main contributions to the literature:

- We argue for the importance of serious games to increase the acceptance of AAL and its enabling technologies;

- We systematically describe the design of Smart Tales, a game that focuses on increasing awareness on AAL in the context of smart homes;

- We present results from a formative evaluation with users, which shows promising results concerning the learning process, but also the need of improving engagement.

The remainder of the paper is structured as follows: Section 2 describes the relevant related work on the use of serious games and virtual worlds in AAL, healthcare, simulation and scenario visualization; Section 3 explains the goals of Smart Tales and describes its design using the Serious Games Design Assessment framework [20]; Section 4 reports on our evaluation of Smart Tales in terms of user engagement and learning effectiveness; finally, Section 5 concludes the paper and outlines future directions.

\section{Related Work}

The most common applications of serious games and virtual worlds in AAL concern motor and cognitive rehabilitation [24] and the improvement of social inclusion [3]. For example, Simmonds and Zikos [26] employ computer games in therapies for pain management, and their evaluation of two games shows improved pain threshold, mood and physical performance.

Game elements and virtual environments can be used for simulation purposes [25], in order to generate data to test artificial intelligence algorithms, e.g., for activity and behavior recognition. Godsey and Skubic [9] propose to simulate sensor networks using game engines, with the aim to reproduce data about the movements of a patient in a home environment. Exploiting the collision mechanism of the physics engine, the authors simulate passive infrared (PIR) sensors and test the correspondence of such a simulation with a real case.

A more advanced framework to collect data about patients' behavior is that by Kormányos and Pataki [13], which features three distinct models for the human, the environment and the motion sensor network. Their simulation generates logs about different variables (bed pressure, unwashed dishes in the sink, etc.) to train and test activity recognition algorithms. Zancanaro et al. [34] propose the SIMDOMO simulator of sensorized domestic environments to generate long-term data concerning human behavior. 
Virtual worlds and 3D environments are applied also during the design, the engineering and the validation of AAL platforms. In designing AAL systems, the use of Interactive Scenario Visualization (ISV) based on 3D models can lead to the clarification of system functionalities, as well as to gain stakeholders' feedback [12]. FernándezLlatas et al. [7] present a computer-aided tool to validate AAL platforms during design, which allows usability engineers to define the workflow of a simulation and to visualize the simulation in a 3D environment.

Smart Tales takes an orthogonal approach: its innovative soul is to use computer serious games to increase end-users' awareness towards ICT solutions for AAL. Smart Tales makes a step forward in the promising research direction of serious gaming to foster acceptance of smart homes, trying to break the learning barriers in the field [15].

\section{Smart Tales}

Smart Tales is a serious game that aims to raise awareness about the potential of Information \& Communication Technology (ICT) solutions for AAL. The purpose of the game is to give the opportunity to end-users to learn about sensors that can be placed in a smart home, showing their capabilities as well as their weaknesses. The version of the game described in this paper is focused on motion detection and indoor positioning.

The player is the inhabitant of a smart home that is equipped with a number of sensors to detect the presence of the player's avatar, and to determine the room where the player is located. Three kinds of sensors are present in the virtual world: passive infrared (PIR) sensors for motion detection; active infrared sensors for passage detection; smart tiles for both motion and presence detection [22].

To engage the player, Smart Tales includes elements from different game genres: third/first person shooters, puzzle games and arcade games. The virtual environment of the game is in 3D (inside a dwelling) and the player controls an avatar through the arrow keys. The goals are to avoid being detected by the sensors in order to score points, and to progress to the next levels while gathering information about AAL and sensors. The player's goal is to provide evidence that some of the sensors are misplaced. In other words, the player is supposed to cheat the home, scoring points as much as he can. The game consist of levels: upon completing a level, the following level is unlocked.

The implementation relies on the Unity 3D (www.unity3d.com/) 4.6 game engine; 3D models of the smart home were built using Blender (www.blender.org/) and SweetHome3D (www.sweethome3d.com/).

\subsection{Game Purpose}

Being a serious game, the main goal of Smart Tales goes beyond pure entertainment [33]: the game is intended as a driver to achieve wider awareness and acceptance of ICT solutions for AAL by end-users. Thus, engagement and effectiveness of learning are the key determinants to assess the success of the game. To achieve such purpose, the design of Smart Tales takes into account multiple, partially conflicting requirements:

- Both technical and non-technical people need to be able to play the game. Hence, the controls should be easy to remember, minimal and intuitive; 
- Information about AAL shall be delivered within the game mechanics in order to make the learning process lighter, using typical game features (such as providing challenges and goals, stimulating curiosity, cooperation, competition) to enhance user engagement and intrinsic motivation [17];

- The player shall be motivated to keep playing through all the levels, avoiding to overwhelm him with excessive information. Moreover, in line with the theory of flow [5] the game difficulty has to gradually increase, increasing the challenge but not the frustration, in order to reward the player for his progress and his learning.

The design of Smart Tales directly addresses its main purpose and these requirements, as explained in the next subsection.

\subsection{Game Design}

To describe the design of Smart Tales in a systematic manner we use the Serious Game Design Assessment (SGDA) framework, proposed by Mitgutsch and Alvarado [20]. This framework enables studying a serious game design in relation to the game purposes and intention: it allows to analyze a serious game investigating the cohesiveness of its content and information, mechanics, fiction and narrative, aesthetics and graphics and framing, and their coherence in relation to the game purpose.

Content \& Information. In the SDGA framework, this aspect refers to the information and data provided during the game. We can distinguish between $a$ ) the serious content that provides information about AAL and the sensors present in the virtual home, and $b)$ the information related to the game status.

The serious content relates to the core purpose of Smart Tales: in the game, the player has to collect informative cards (badges) about Ambient Assisted Living and the sensors present in the game levels. The player can read the collected badges in any moment of the game except while he is answering the quizzes at the end of each level, for the provided information is needed to correctly answer to the questions.

Figure 1 shows the information in one of the badges, that defines the notion of a "smart tile". The provided information is both textual and visual. The textual information describes the content of the badge, while the visual information facilitates the recognition of the badge. The top-left "information logo" on the badge clarifies that the included information is useful to answer to the questions at the end of the level; the top-right logo identifies the main topic of the badge: there are different logos for badges on general information about AAL, PIR sensors, active infrared sensors and smart tiles.

In order to keep the player engaged in the game, to not overwhelm him with too extensive information and to make the learning process more effective, the information is split in nine cards. Furthermore, to ease the reading process, the content of the badges with longer content is presented in multiple pages.

During the game, the player can visualize data about the game status, including the time left to play the level, the earned points (and the points of his opponent, the engineer who configured the smart home, covered by the $\mathrm{CPU}$ ) and the number of unread badges in the player's inventory. As depicted in Figure 2, the player gets notified whenever he scores points. Such elements allow to keep the player focused on his goals in the game, 


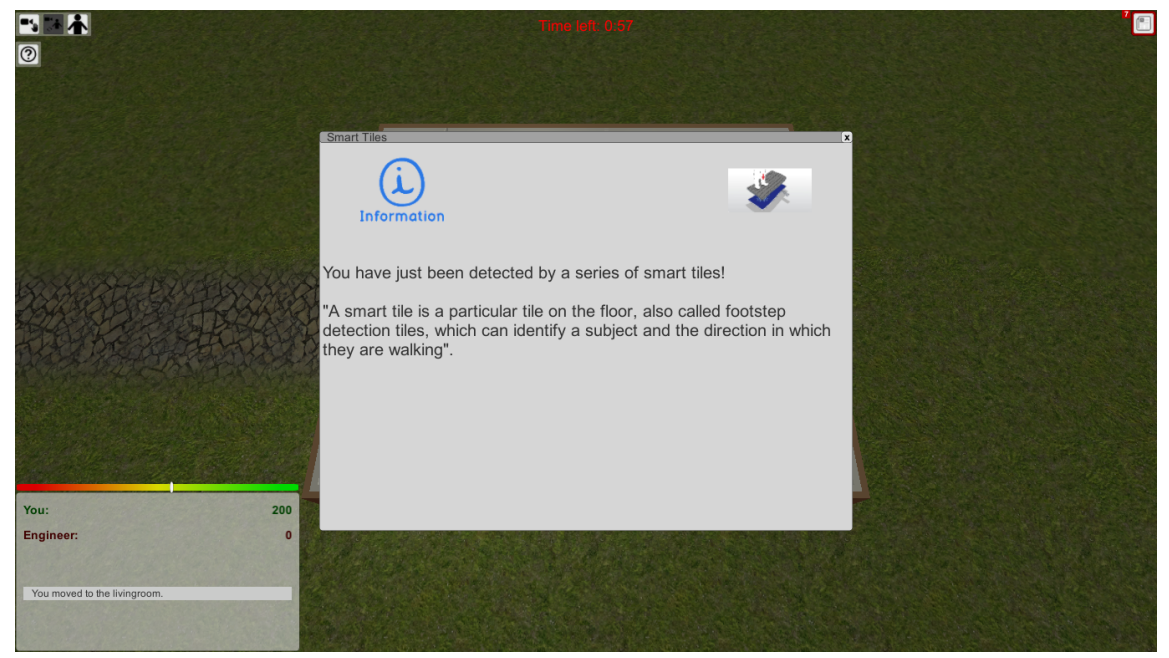

Fig. 1. Delivering awareness through badges: the definition of the sensor "smart tile".

pushing him to beat the home engineer, collecting and reading more badges, in order to foster engagement as a key factor in the learning process.

Game Mechanics. Mechanics are the various actions, behaviors and control mechanisms afforded to the player within a game context [11]. In the SGDA framework, the game mechanics involve the in-game goal of the game, the reward system, and the main playful obstacles/challenges. In Smart Tales, in order to advance to the next level, the player has to achieve three goals:

- Score more points than the home engineer;

- Collect all the badges within the level;

- Answer correctly to all the questions presented at the end of a level.

The first goal gives a challenge to the player: he needs to beat the engineer who configured the home. The difficulty increases with the levels, and the player has to carefully plan an effective path for his avatar to follow. To do so, the player can choose in which room he wants to place his avatar; some choices are better than others, and the right positioning can be crucial to pass the level. Thus, challenge and planning are instrumental to keep the player focused and engaged. The second goal pushes the player to collect all the badges with information about AAL. Finally, to unlock the next level, and thus to continue the challenge, the player needs to answer to three questions at the end of each level. The content in the badges is essential to answer correctly. In case wrong answers are given, the player does not unlock the next level.

To score points, the player needs to exit from the rooms of the smart home without being detected. When a sensor detects the presence of a player inside a room, the home engineer scores points. Consecutive actions give extra-bonus points, using a multiplier, as in arcade games, either to the player for having cheated the house, or to the CPU engineer for having kept track of the player. The basic action that the player can perform is to move his avatar in the 3D smart home, using the arrow keys, in order to avoid 


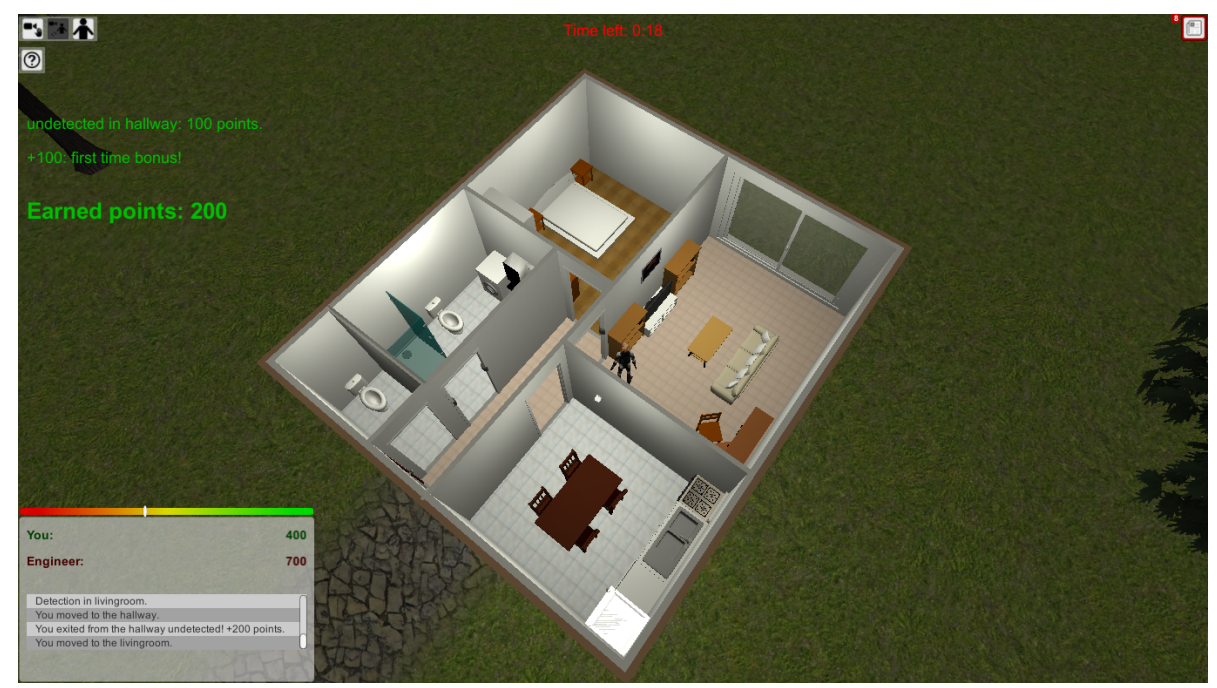

Fig. 2. In-game notification to the player: by exiting the hallway undetected for the first time in that level, the player scores 100 points.

the placed sensors. The player is able to see the sensors' coverage before placing the avatar in the home; while playing, he can see the devices on the walls and doors, but not their sensing area. Moreover, the player gets bonus points by reading the informative badges: this mechanism gives an extra in-game incentive to read the AAL-related content. Another game mechanic is the time limit to play a level, for the player has one minute to complete the level and to achieve the first two objectives: to score more than the engineer and to collect all the badges. On the one hand, the time limit makes the level more challenging; on the other hand, keeping it low allows the player to not waste time waiting the quiz at the end of the level, in case he achieves the first two goals.

Fiction \& Narrative. The fictional context of Smart Tales has the following logline: an engineer equipped an assistive "unobtrusive" environment (a smart home), but the skeptical inhabitant does not consider the home that unobtrusive. The player lives in the home and has to prove that the engineer has misplaced the sensors. The game takes place in a dwelling: the player has to place his avatar in a room and then he has to start moving, collecting badges and avoiding sensors.

The fictional context reflects the target environment of AAL: the home of an assisted person. The narrative introduces the player to the game challenges that will engage him in his learning process: the inhabitant does not consider the home unobtrusive, and his aim is to mislead sensors to proof they are misplaced.

Aesthetics \& Graphics. To support the game purpose, Smart Tales uses basic 3D graphics to represent the smart home and the players avatar. A 3D environment gives additional opportunities to the player to avoid the sensors: in addition to moving inside the home, the avatar can walk crouched, jump and do small steps. To support the game mechanics, the main elements are highlighted in the user interface: the time is shown 
in red color on the top-center of the screen; the scores are on the bottom left of the screen (the player points are in green, the engineer points are in red). In addition, as per Figure 2, a status bar shows whether the player is currently defeating the engineer, by positioning a cursor within a red-to-green scale (red = engineer is leading, green = player is leading). A green notification on the left side of the screen appears when the player scores points; a similar red-colored notification appears when the home engineer scores points on the right side of the screen. Finally, a set of icons allows the player to change the game camera (top-left of the screen) and to access the inventory of the collected badges (top right of the screen).

Framing. This element of the SGDA framework refers to the target group of a serious game. Since Smart Tales' purpose is to increase the awareness of ICT solutions for AAL, the target group is composed by people potentially interested in equipping a home with smart technology, such as the relatives of older adults who live alone. Hence, Smart Tales is mainly targeted to non-technical people and non-gamers. The complexity of the levels gradually increases in order to not overwhelm the player. In the first level, a few PIR sensors are available, and the badges are easy to collect. In the third and last level, both passive and active infrared sensors as well as smart tiles are present, and the player has properly plan beforehand on how to collect the badges, bearing in mind that he should score more points than the home engineer.

The controls of the game are easy to allow non-technical people to play: the avatar can be moved with the arrow keys (or the "WASD" keys) and the buttons on the screen are mouse-clickable. The default game camera is automatic and follows the avatars' movements, from a third person perspective. However, more skilled players can choose a manual camera that can be rotated with the mouse and can even use the "CTRL" key to make the avatar crouch, the "Q" key to do just a small step and the "space bar" to jump. A detailed tutorial explains all the controls of the game. As a reminder of the game controls, the player can always press the "question mark" button on the top-left of the screen: it shows the game controls and summarizes the level's goals.

\section{Evaluation}

We report on a preliminary evaluation concerning user engagement and learning effectiveness. Engagement is key to keep the player motivated to play, so that the entire (or at least most of the) gameplay is experienced. This is important because the serious purpose of the game-raising awareness on AAL - is delivered through the gameplay by collecting badges and answering to questions.

Our evaluation method involved user tests with ten people having different backgrounds, selected because of their inexperience in AAL. All the participants were from the same geographical area: the Netherlands. Eight participants were male and two were female. Nine of them had university level education in various fields of expertise including Mathematics, Psychology, Linguistics, Human/Economic Geography, Anthropology, Social Studies, Film Sciences, History and Electrical Engineering. One had secondary school education level. The age ranged from 21 to 30 years old. All but one finished the game and completed all the three levels of the tested version of Smart Tales. 
We based the tests on the quasi-experimental research design model of Mayer et al. [19], and our study was conducted by three M.Sc. students. After an explanation of the game goals and of the evaluation's objectives, the participants filled in a short questionnaire to determine their prior knowledge on AAL and the sensors that Smart Tales features. Then, the participants were asked to play the three levels of the game, without imposing time limits. In the pre-game questionnaire, the users had to give yes/no answers to the following questions:

1. Do you know what is ambient assisted living?

2. Do you know what is a smart home?

3. Do you know what is a passive infrared sensor?

4. Do you know what is an active infrared sensor?

5. Do you know what is a smart tile?

As a double check, the questionnaire included five open questions that asked the participant an informal definition of the concepts of the previous five questions. Seven participants answered negatively to all of the questions; however, when asked to define AAL using their own words, they associated it to the concept of a "home technologically equipped to assist in some task and/or perform monitoring". One participant (with a background in Electrical Engineering) answered positively to the first four question, although he gave a wrong definition of infrared sensors. The last two participants knew what is a smart home; one of them answered "yes" on the last two questions, but actually gave a wrong definition of active infrared sensor and smart tile.

After the gameplay session, the participants had to fill in two questionnaires: the former measured user engagement, and was based on the User Engagement Scale (UES) proposed by Wiebe et al. [32] to assess engagement in video game-based environments; the latter focused on the assimilated knowledge by asking the same questions that were in the game, and was delivered thirty minutes after the end of the game session. The choice of relying on the same questions poses threats to the validity of the results, while it minimizes the chance of asking knowledge that the game did not deliver.

The tests were carried out in two sessions, at different stages of game development. Six participants tested an early version of the game, while four participants tested a revised version, including different textual and visual information. The early version displayed information on the badges without visual logos. Moreover, a clearer description of the in-game goals was added before the first level as well as in the game help. We performed this redesign after collecting feedback from the first six participants, in order to improve usability and limit confusion. In Section 4.2, we highlight the different results from the two test sessions.

\subsection{Learning}

In Smart Tales, the player has to answer three questions at the end of each level, in order to advance to the next one. The questions concern the serious information content that is contained in the badges collected in the level that was just played. The choice of including knowledge into artifacts is inspired by the reified knowledge game design pattern [18], in order to introduce knowledge into a game without disrupting the flow. 


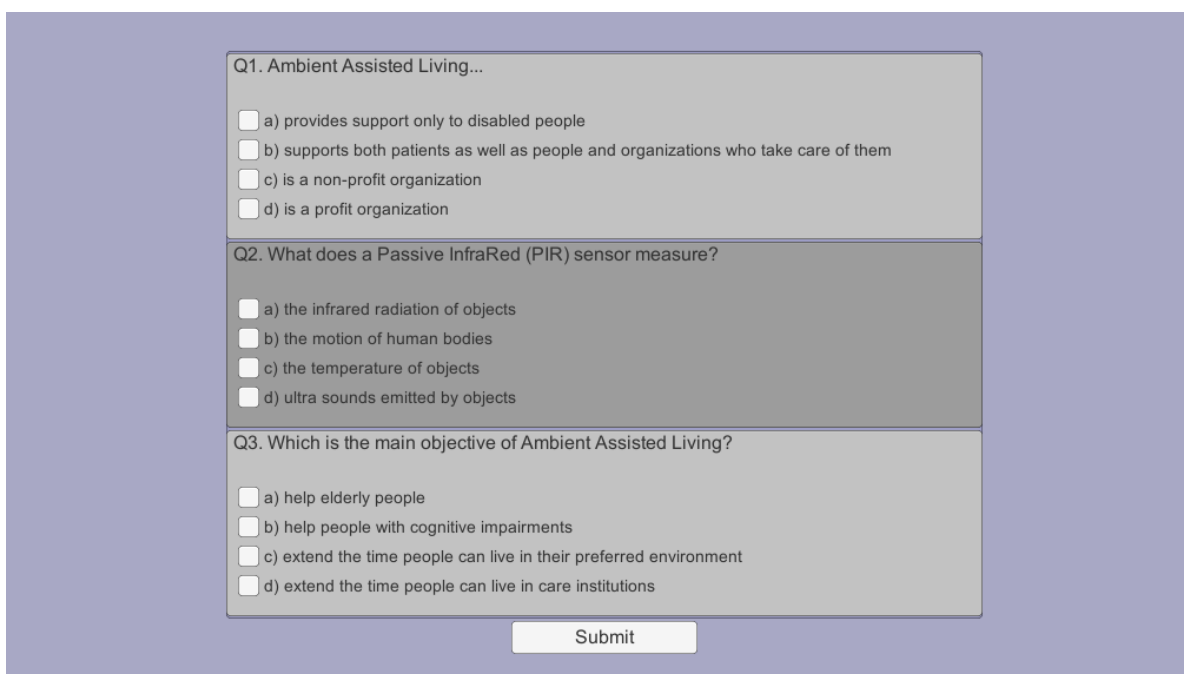

Fig. 3. The questions the player is confronted with at the end of the first level to unlock level two.

Figure 3 shows the questions at the end of the first level. These questions check the acquired knowledge on the concept of AAL and on the definition of PIR sensor; such topics are covered by the badges placed in the first level. While answering the in-game questions, the player is actually performing a self-assessment of his first-order learning. Visual feedback informs the player about the correctness of his answers: those highlighted in green are correct, while those in red are wrong (see Figure 4). If wrong answers are given, the player has to replay the level and re-answer the questions, in order to unlock the next level. This is an incentive for learning the content in the badges.

During the users tests, we evaluated first-order learning: according to Mayer et al. [19], it is direct influence of playing the game on the individual, small group attitudes, knowledge, skills or behavior. We reused the nine questions that the player has to answer while playing Smart Tales (three per level): the questions were provided to the users as a questionnaire, thirty minutes after playing the game, in order to assess the effectiveness of the learning process. Table 1 includes the number of correct answers per player in the post-game questionnaire. Globally, $81 \%$ of the answers given by the participants was correct: one player answered all the questions correctly, while five players gave just one wrong answer. However, one player was able to give only four correct answers. In fact, such player was not able to complete the second level, and gave up after five attempts; this means that he did not read all the badges and he did not learn enough to answer to more questions.

The approach that we employed in our preliminary evaluation of learning effectiveness does inevitably suffer from threats to validity:

- To adequately assess the learned knowledge, the time between the end of the game session and the post-questionnaire should be longer than thirty minutes. Further tests with an improved research protocol are essential; 


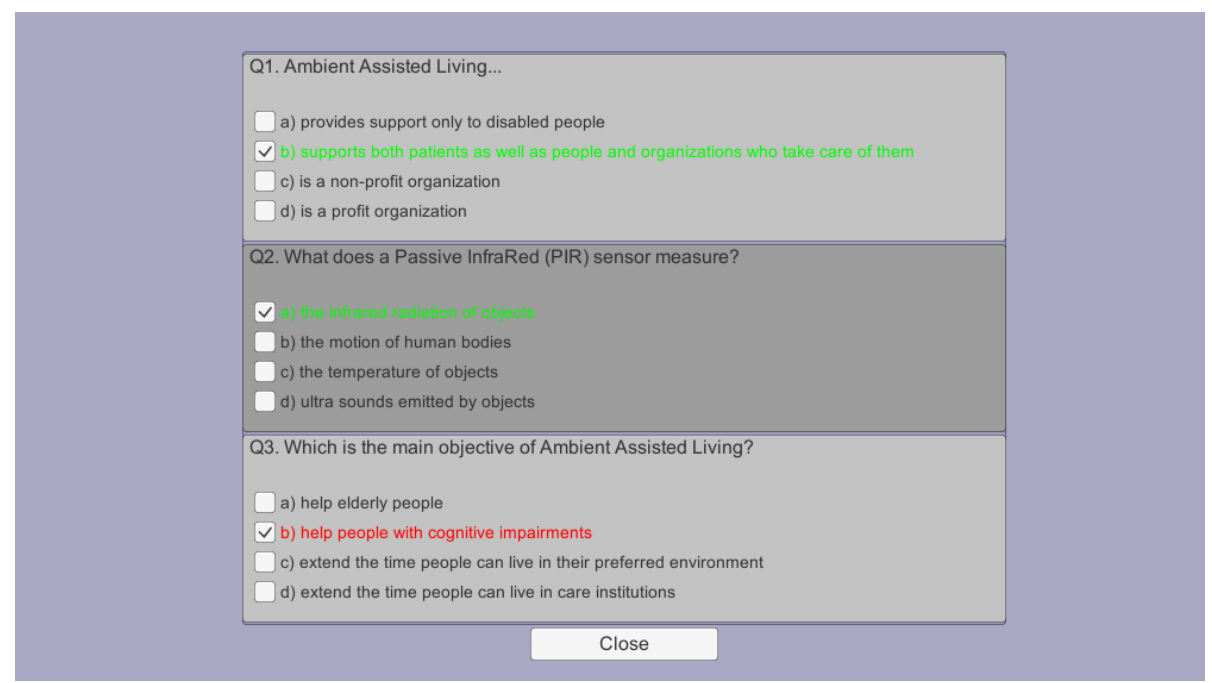

Fig. 4. The answers given by the player: the game provides a visual feedback on the correct answers (green) and on the wrong ones (red).

Table 1. Number of correct answers per player in the post-game questionnaire.

\begin{tabular}{ll|ll}
\hline Player 1 & $6 / 9$ & Player 6 & $4 / 9$ \\
\hline Player 2 & $8 / 9$ & Player 7 & $8 / 9$ \\
\hline Player 3 & $8 / 9$ & Player 8 & $7 / 9$ \\
\hline Player 4 & $9 / 9$ & Player 9 & $8 / 9$ \\
\hline Player 5 & $7 / 9$ & Player 10 & $8 / 9$ \\
\hline
\end{tabular}

- A comparison with traditional learning methods (for example reading books or papers) should be performed, in order to clearly evaluate whether our awareness game is suitable for teaching the basics of AAL and its enabling technologies. This should involve a different research method where participants are allocated to either a treatment group (playing the serious game) or a control group (relying on other instructional techniques) [1].

- The post-questionnaire should be slightly different from the in-game questions, in order to be sure that the tester does not memorize the correct answer, but instead learns the information provided by the badges.

\subsection{Engagement}

To evaluate user engagement in Smart Tales, we used the User Engagement Scale (UES) proposed by Wiebe et al. [32] as a validated measure that is tailored for videogames. According to the UES, engagement is composed by four factors:

- Focused attention, based on the theory of flow [5], to evaluate the concentration, the temporal dissociation and the absorption of the player; 
- Perceived usability, to evaluate affective (challenge/frustration) and cognitive (effortful) aspects of playing the game;

- Aesthetics, to evaluate the visual appearance of the game;

- Satisfaction, to evaluate the interest the game evoked, the fun of the gaming experience and the willingness to play it again (and to recommend the game to others).

The score each factor, we posed statements that the users could agree or disagree with using a 5-point Likert scale, where 1 corresponds to strong disagreement and 5 to strong agreement with the statement. In order to compute a score for each factor, we used the average of statements that related with that factor.

Table 2 shows the UES scores obtained by computing the mean of scores given by the participants, before and after the redesign phase. The score for focused attention was 2.42 before the redesign phase and 2.53 after the redesign $(1=$ low ability to focus on the game, $5=$ high ability to focus). The perceived usability scored 3.4 before and 1.91 after $(1=$ high sense of usability, $5=$ low sense of usability); the aesthetics scored 2.8 before and 2.9 after ( $1=$ low appealing game, $5=$ high appealing game). We combined these factors into the global satisfaction value, which corresponds to 2.64 before and 2.04 after $(1=$ low satisfaction, $5=$ high satisfaction $)$. The data highlights a significant improvement in the perceived usability, and a slight improvement in the focused attention and in the aesthetics, and thus a more engaging game. Nevertheless, user satisfaction was lower after the redesign: this can be due to the fact that the three ingame goals were explicitly listed at the beginning of the level, instead of being present just in the tutorial; hence, the player might feel overwhelmed by the game goals. The small number of subjects, however, does not allow to draw any statistically significant conclusion. Each factor is analyzed individually below.

Table 2. UES results for Smart Tales, before and after the minor redesign.

\begin{tabular}{lc|c}
\hline UES factor & Before & After \\
\hline \hline Focused Attention & 2.42 & 2.53 \\
\hline Perceived Usability & 3.40 & 1.91 \\
\hline Aesthetics & 2.80 & 2.90 \\
\hline Satisfaction & 2.64 & 2.04 \\
\hline
\end{tabular}

Focused Attention. Table 3 lists the statements associated with the factor "Focused Attention". Before the performed redesign, all the statements scored between weak disagreement (2) and neutrality (3). The statements with the lowest score are those about the perception of time, while the statements about absorption and concentration got a score closer to neutrality (i.e., 2.67 and 2.83). While players need to concentrate to achieve the game goals, they seem not to lose awareness of the fact that they are performing a learning task. The redesign seems to have an impact on player absorption: the statement $I$ was absorbed in my gaming task went from a value between weak disagreement and neutrality (2.33) to a value close to weak agreement (3.75); moreover, 
such impact is confirmed by the standard deviation of the answers, which diminished significantly with the redesign.

Table 3. The average scores of the statements concerning focused attention $(\bar{x})$, before and after the minor redesign, as well as the related standard deviation $(\sigma)$.

\begin{tabular}{|l|c|c|c|c|}
\hline Focused attention statement & \multicolumn{2}{|c|}{ Before } & \multicolumn{2}{|c|}{ After } \\
\hline When I was playing the game I lost track of the world around me. & 2.50 & 1.12 & 2.50 & 0.50 \\
\hline I blocked out things around me when I was playing the game. & 2.67 & 1.25 & 3.00 & 0.71 \\
\hline The time I spent playing the game just slipped away. & 2.17 & 1.07 & 2.25 & 0.43 \\
\hline I was absorbed in my gaming task. & 2.33 & 1.11 & 3.75 & 0.43 \\
\hline I was so involved in my gaming task that I lost track of time. & 2.00 & 1.00 & 1.50 & 0.50 \\
\hline During this gaming experience I let myself go. & 2.83 & 1.34 & 2.00 & 0.71 \\
\hline I lost myself in this gaming experience. & 2.33 & 1.11 & 1.75 & 0.43 \\
\hline I was really drawn into my gaming task. & 2.50 & 0.50 & 3.50 & 0.50 \\
\hline
\end{tabular}

Perceived Usability. Table 4 includes the statements related to the factor "Perceived Usability": the mean of the scores before the redesign is in between neutrality (3) and weak disagreement (2), thereby calling for improving usability of the game. In particular, the participants felt frustrated: all the players but one weakly agreed with the sentence I felt frustrated while playing the game ( $\sigma=0.37$ ).

Follow-up interviews with the users revealed that the main reason was that players did not really know what to do due to ambiguous or missing feedback and a lack of instructions. The tutorial of Smart Tales includes written instructions given to the player using pop-ups. Most of the players found such instruction boring, and skipped the pop-ups before reading the content. Thus, a redesign might be needed, and tests with different techniques to give instructions should be carried out (for example we can consider to give audio instructions and to use subtitles).

However, as highlighted at the beginning of Section 4.2, the results on perceived usability after the redesign are encouraging: each statement gets a better score, and perceived usability globally scores 1.91 . The sense of frustration was lower and all the participants agreed that they were able to do the things they needed to do in the game.

Aestethics. Table 5 shows the scores of the statements on factor "Aestethics". Users gave a positive score-between neutrality (3) and weak agreement (4) - to the statements on layout and graphics, which indicates that the basic graphics chosen for Smart Tales are appropriate for the purpose of the game. Nevertheless, the users did not define the game as very attractive (the statement on game attractiveness scored between weak disagreement and neutrality) since the graphics reminds third person shooter games, which typically have high-quality visual effects. This expectation can have a negative impact on global engagement: the statement the game was aesthetically appealing 
Table 4. The average scores of the statements concerning perceived usability $(\bar{x})$, before and after the minor redesign, as well as the related standard deviation $(\sigma)$.

\begin{tabular}{|l|c|c|c|c|}
\hline Perceived usability & \multicolumn{2}{|c|}{ Before } & \multicolumn{2}{|c|}{ After } \\
& $\bar{x}$ & $\sigma$ & $\bar{x}$ & $\sigma$ \\
\hline \hline I felt discouraged while playing the game. & 3.83 & 1.50 & 1.07 & 0.50 \\
\hline I felt annoyed while playing the game. & 3.67 & 2.25 & 0.94 & 0.43 \\
\hline Playing the game was mentally taxing. & 2.83 & 1.75 & 0.69 & 0.43 \\
\hline I found the game confusing to use. & 3.83 & 2.50 & 1.07 & 0.50 \\
\hline I felt frustrated while playing the game. & 3.83 & 1.50 & 0.37 & 0.50 \\
\hline I could not do some of the things I needed to do in the game. & 2.83 & 1.00 & 1.21 & 0.00 \\
\hline The gaming experience was demanding. & 3.33 & 2.50 & 0.75 & 0.50 \\
\hline This gaming experience did not work out the way I had planned. & 3.00 & 2.25 & 1.15 & 0.43 \\
\hline
\end{tabular}

scored 2.67 before the redesign and 2.25 after the redesign; most of the participants (6 out of 10) weakly disagreed with the sentence, three were neutral and one agreed.

Table 5. The average scores of the statements concerning aesthetics $(\bar{x})$, before and after the minor redesign, as well as the related standard deviation $(\sigma)$.

\begin{tabular}{|l|c|c|c|c|}
\hline \multirow{2}{*}{ Aestethic statement } & \multicolumn{2}{|c|}{ Before } & \multicolumn{2}{|c|}{ After } \\
& $\bar{x}$ & $\sigma$ & $\bar{x}$ & $\sigma$ \\
\hline \hline Iliked the graphics and images used in the game. & 3.17 & 4.00 & 1.34 & 0.71 \\
\hline The game appealed to my visual senses. & 2.50 & 2.75 & 1.26 & 0.83 \\
\hline The game was aesthetically appealing. & 2.67 & 2.25 & 0.75 & 0.43 \\
\hline The screen layout of the game was visually pleasing. & 2.83 & 3.50 & 0.90 & 1.12 \\
\hline The game was attractive. & 2.83 & 2.00 & 0.90 & 0.71 \\
\hline
\end{tabular}

Satisfaction. Table 6 includes the scores of the statements about factor "Satisfaction". Both before and after the redesign phase, the players indicated a low willingness to continue playing and to suggest the game to other people. In addition, all the participants to the tests performed after the redesign weakly disagreed with the sentence Playing the game was worthwhile. One reason for these issues is that the same home and sensors are present in all the three levels; thus, some players might find the game repetitive. Hence, a redesign might be needed, by adding levels with different home maps and more sensor types. The new redesign should address also players' curiosity and interest, since the participants were neutral toward these two aspect of the serious game.

\section{Discussion and Future Directions}

In this paper, we presented the Smart Tales game that raises awareness on concept, objectives, working principles and enabling technologies of ambient assisted living 
Table 6. The average scores of the statements concerning satisfaction $(\bar{x})$, before and after the minor redesign, as well as the related standard deviation $(\sigma)$.

\begin{tabular}{|l|c|c|c|c|}
\hline Satisfaction statement & \multicolumn{3}{|c|}{ Before } & \multicolumn{2}{|c|}{ After } \\
& $\bar{x}$ & $\sigma$ & $\bar{x}$ & $\sigma$ \\
\hline The content of the game incited my curiosity. & 3.33 & 2.50 & 0.94 & 1.12 \\
\hline I would continue to play this game out of curiosity. & 2.00 & 1.25 & 1.00 & 0.43 \\
\hline I would recommend playing the game to my friends and family. & 2.17 & 1.75 & 1.21 & 0.43 \\
\hline Playing the game was worthwhile. & 2.33 & 2.00 & 1.25 & 0.00 \\
\hline I felt interested in my gaming task. & 3.00 & 2.50 & 1.15 & 0.50 \\
\hline My gaming experience was rewarding. & 2.33 & 2.00 & 0.94 & 0.71 \\
\hline This gaming experience was fun. & 3.33 & 2.25 & 0.94 & 0.43 \\
\hline
\end{tabular}

(AAL). Currently, the game has three levels as well as a tutorial for the user to learn about the game controls and objectives. The player impersonates an inhabitant of a smart home, and has to cheat the deployed sensors. While doing so, the player has also to collect informative badges about AAL and its technology, gaining the necessary knowledge to answer the quizzes at the end of each level, and advance to the next one.

We conducted a preliminary formative evaluation on learning effectiveness and user engagement with ten participants. Six participants played the game in its early stage, while the other four played an improved version, where we performed a minor redesign of the informative badges. The results concerning the learning process are promising and encourage the use of serious games to increase the end-users' knowledge on AAL and its enabling technologies: in the posttest questionnaires, $81 \%$ of the answers were correct. Nevertheless, more extensive tests need to conducted: a comparison with a control group relying on traditional learning techniques can better validate the usefulness and effectiveness of serious games to increase awareness about AAL.

The tests on user engagement, based on the UES revised by Wiebe et al. [32], show clear room for improvement. On the one hand, the redesigned version obtained a perceived usability score of 1.91 in a scale from 1 to 5 (where 1 represents a high sense of usability and 5 a low sense of usability). On the other hand, focused attention, aesthetics and satisfaction obtained an average score at the middle of the scale: the repetitiveness of the levels needs to be reduced (e.g., by creating levels with different home maps and adding new sensors), to increase the players' curiosity and sense of fun; improving the graphics could lead to higher immersion and, thus, enhance the sense of flow.

Future work includes improving Smart Tales based on the outcomes of the formative evaluation, and to conduct further tests with users. Another direction concerns replacing the CPU-as-an-engineer gameplay with an additional role for the players, i.e., that of an engineer that has to properly configure a smart home. Future versions should also recognize that $\mathrm{AAL}$ and smart homes define socio-technical systems [6], rather than focusing only on the technological infrastructure.

Acknowledgments. The authors thank Brian Arendse, Mats Hofman and Lamia Soussi for their precious help in carrying out the users tests of Smart Tales. 


\section{References}

1. Bellotti, F., Kapralos, B., Lee, K., Moreno-Ger, P., Berta, R.: Assessment in and of serious games: An overview. Advances in Human-Computer Interaction 2013, 1:1-1:11 (2013)

2. Ben Allouch, S., van Dijk, J., Peters, O.: The acceptance of domestic ambient intelligence appliances by prospective users. In: Tokuda, H., Beigl, M., Friday, A., Brush, A., Tobe, Y. (eds.) Pervasive Computing, LNCS, vol. 5538, pp. 77-94. Springer Berlin Heidelberg (2009)

3. Cascado, D., Romero, S., Hors, S., Brasero, A., Fernandez-Luque, L., Sevillano, J.: Virtual worlds to enhance ambient-assisted living. In: Engineering in Medicine and Biology Society (EMBC), 2010 Annual International Conference of the IEEE. pp. 212-215 (2010)

4. Coughlin, J., D’Ambrosio, L., Reimer, B., Pratt, M.: Older adult perceptions of smart home technologies: Implications for research, policy \& market innovations in healthcare. In: Engineering in Medicine and Biology Society, 2007. EMBS 2007. 29th Annual International Conference of the IEEE. pp. 1810-1815 (2007)

5. Csikszentmihalyi, M.: Flow and the Psychology of Discovery and Invention. HarperPerennial, New York (1997)

6. Dalpiaz, F., Giorgini, P., Mylopoulos, J.: Adaptive Socio-Technical Systems: a Requirements-driven Approach. Requirements Engineering 18(1), 1-24 (2013)

7. Fernandez-Llatas, C., Mocholi, J., Sala, P., Naranjo, J., Pileggi, S., Guillen, S., Traver, V.: Ambient assisted living spaces validation by services and devices simulation. In: Engineering in Medicine and Biology Society, EMBC, 2011 Annual International Conference of the IEEE. pp. 1785-1788 (2011)

8. Gaul, S., Ziefle, M.: Smart home technologies: Insights into generation-specific acceptance motives. In: Holzinger, A., Miesenberger, K. (eds.) HCI and Usability for e-Inclusion, LNCS, vol. 5889, pp. 312-332. Springer Berlin Heidelberg (2009)

9. Godsey, C., Skubic, M.: Using elements of game engine architecture to simulate sensor networks for eldercare. In: Engineering in Medicine and Biology Society, 2009. EMBC 2009. Annual International Conference of the IEEE. pp. 6143-6146 (2009)

10. Hildmann, H., Hirsch, B.: Raising awareness for environmental issues through mobile device based serious games. 4th Microsoft Academic Days, Berlin, Germany (2008)

11. Hunicke, R., Leblanc, M., Zubek, R.: MDA: A formal approach to game design and game research. In: Proceedings of the AAAI Challenges in Game AI Workshop. AAAI Workshop - Technical Report, vol. WS-04-04, pp. 1-5 (2004)

12. van't Klooster, J.W., van Beijnum, B.J., Eliens, A., Hermens, H.: Interactive scenario visualization for user-based service development. In: Collaboration Technologies and Systems (CTS), 2012 International Conference on. pp. 498-503 (2012)

13. Kormányos, B., Pataki, B.: Multilevel simulation of daily activities: Why and how? In: Computational Intelligence and Virtual Environments for Measurement Systems and Applications (CIVEMSA), 2013 IEEE International Conference on. pp. 1-6 (2013)

14. Lee, C., Coughlin, J.F.: Perspective: Older adults' adoption of technology: An integrated approach to identifying determinants and barriers. Journal of Product Innovation Management 32(5), 747-759 (2015)

15. Leonhardt, S., Kassel, S., Randow, A., Teich, T.: Learning in the context of an ambient assisted living apartment: Including methods of serious gaming. In: Motta, G., Wu, B. (eds.) Software Engineering Education for a Global E-Service Economy, pp. 49-55. Progress in IS, Springer International Publishing (2014)

16. Lutz, W., Sanderson, W., Scherbov, S.: The coming acceleration of global population ageing. Nature 451(7179), 716-719 (2008)

17. Malone, T.W., Lepper, M.R.: Making learning fun: A taxonomy of intrinsic motivations for learning. In: Snow, R., Farr, M.J. (eds.) Aptitude, Learning, and Instruction Volume 3: Conative and Affective Process Analyses, pp. 223-253. Lawrence Erlabaum Associates (1987) 
18. Marne, B., Wisdom, J., Huynh-Kim-Bang, B., Labat, J.M.: The six facets of serious game design: A methodology enhanced by our design pattern library. In: Ravenscroft, A., Lindstaedt, S., Kloos, C., Hernndez-Leo, D. (eds.) 21st Century Learning for 21 st Century Skills, LNCS, vol. 7563, pp. 208-221. Springer Berlin Heidelberg (2012)

19. Mayer, I., Bekebrede, G., Harteveld, C., Warmelink, H., Zhou, Q., van Ruijven, T., Lo, J., Kortmann, R., Wenzler, I.: The research and evaluation of serious games: Toward a comprehensive methodology. British Journal of Educational Technology 45(3), 502-527 (2014)

20. Mitgutsch, K., Alvarado, N.: Purposeful by design? A serious game design assessment framework. In: Proceedings of the International Conference on the Foundations of Digital Games - FDG '12. pp. 121-128. ACM Press (2012)

21. Newbould, M., Furnell, S.: Playing safe: A prototype game for raising awareness of social engineering. In: Australian Information Security Management Conference. pp. 24-30 (2009)

22. Rashidi, P., Mihailidis, A.: A survey on ambient-assisted living tools for older adults. Biomedical and Health Informatics, IEEE Journal of 17(3), 579-590 (2013)

23. Rebolledo-Mendez, G., Avramides, K., de Freitas, S., Memarzia, K.: Societal impact of a serious game on raising public awareness: the case of floodsim. In: Proceedings of the 2009 ACM SIGGRAPH Symposium on Video Games. pp. 15-22. ACM (2009)

24. Rego, P., Moreira, P., Reis, L.: Serious games for rehabilitation: A survey and a classification towards a taxonomy. In: Information Systems and Technologies (CISTI), 2010 5th Iberian Conference on. pp. 1-6 (2010)

25. Sernani, P., Claudi, A., Calvaresi, P., Accattoli, D., Tofani, R., Dragoni, A.F.: Using 3D simulators for the ambient assisted living. In: 3rd International Workshop on Artificial Intelligence and Assistive Medicine. CEUR Workshop Proceedings, vol. 1213, pp. 16-20 (2014)

26. Simmonds, M.J., Zikos, D.: Computer games to decrease pain and improve mood and movement. In: Proceedings of the 7th International Conference on PErvasive Technologies Related to Assistive Environments. pp. 56:1-56:4. ACM (2014)

27. Steinke, F., Bading, N., Fritsch, T., Simonsen, S.: Factors influencing trust in Ambient Assisted Living Technology: A scenario-based analysis. Gerontechnology 12(2), 81-100 (2014)

28. Sun, H., De Florio, V., Gui, N., Blondia, C.: Promises and challenges of ambient assisted living systems. In: Information Technology: New Generations, 2009. ITNG '09. Sixth International Conference on. pp. 1201-1207 (2009)

29. Susi, T., Johannesson, M., Backlund, P.: Serious games: an overview. Tech. Rep. HS-IKITR-07-001, School of Humanities and Informatics, University of Skövde (2007)

30. Townsend, D., Knoefel, F., Goubran, R.: Privacy versus autonomy: A tradeoff model for smart home monitoring technologies. In: Engineering in Medicine and Biology Society, EMBC, 2011 Annual International Conference of the IEEE. pp. 4749-4752 (2011)

31. Werblow, A., Felder, S., Zweifel, P.: Population ageing and health care expenditure: a school of red herrings? Health Economics 16(10), 1109-1126 (2007)

32. Wiebe, E.N., Lamb, A., Hardy, M., Sharek, D.: Measuring engagement in video game-based environments: Investigation of the user engagement scale. Computers in Human Behavior 32(0), 123-132 (2014)

33. Winn, B.M.: The Design, Play, and Experience Framework. In: Handbook of Research on Effective Electronic Gaming in Education, vol. 5497, pp. 1010-1024. IGI Global (2007)

34. Zancanaro, M., Marchesoni, M., Armellin, G.: SIMDOMO: A tool for long-term simulations of ambient-assisted living. In: Aarts, E., de Ruyter, B., Markopoulos, P., van Loenen, E., Wichert, R., Schouten, B., Terken, J., Van Kranenburg, R., Den Ouden, E., O'Hare, G. (eds.) Ambient Intelligence, LNCS, vol. 8850, pp. 47-50. Springer International Publishing (2014) 